\title{
Surface-wave damping in a brimful circular cylinder
}

\author{
By CARLOS MARTEL, JOSÉ A. NICOLÁS \\ AND JOSÉ M. VEGA \\ Departamento de Fundamentos Matemáticos, Universidad Politécnica de Madrid, \\ Plaza Cardenal Cisneros, 3, 28040 Madrid, Spain
}

The natural frequencies and damping rates of surface waves in a circular cylinder with pinned-end boundary conditions are calculated in terms of the gravitational Reynolds and Bond numbers, $C^{-1}$ and $B$, and the slenderness of the cylinder $A$, in the limit $C \rightarrow 0$. We consider higher-order approximations that include the effect of viscous dissipation in the Stokes boundary layers and the bulk. A comparison with clean-surface experiments by Henderson \& Miles (1994) shows a satifactory agreement except for the first axisymmetric mode, which exhibits a $26 \%$ discrepancy. The much larger dramatic discrepancy of former theoretical predictions is hereby improved and explained.

\section{Introduction}

A precise knowledge of the linear damping rate of surface waves is a prerequisite to constructing a safe and consistent weakly nonlinear theory for the evolution of the waves, which in turn has not been completed to a satisfactory degree in vibrated finite containers (Miles \& Henderson 1990).

The theoretical analysis of linear damping was undertaken by Stokes (1851), who considered an infinite horizontal liquid layer over a flat plate. More recently, several attempts to get precise theoretical predictions in finite containers essentially failed after comparison with experiments. The discrepancy was first assumed to be due to both capillary hysteresis (Benjamin \& Ursell 1954) and surface contamination (Van Dorn 1966 , although surfactants had been recognized as a source of damping much earlier). That led to a subsequent analysis of both effects and to valuable phenomenological boundary conditions to account for them: see Miles (1967) and Henderson \& Miles (1994) for old and new references and for a further discussion on these topics. But more recent experimental measurements in a quite clean brimful circular cylinder with a sharp edge (thus the contact line was essentially fixed and capillary hysteresis was essentially absent) showed again a poor agreement with current theoretical results; an interesting observation was that the discrepancy significantly increased for higher modes. Let us point out here that the difficulty appears also for other shapes of the container (e.g. for rectangular containers, see Henderson \& Miles 1990 and Jiang et al. 1996) and for related fluid configurations such as liquid bridges, where the difficulty was solved by Higuera, Nicolás \& Vega (1994) (see also Higuera \& Nicolás 1997).

The main object of this paper is to precisely calculate the linear damping rate in a finite container. We shall ignore the effects of capillary hysteresis (by fixing 
the contact line) and surface contamination, and we shall restrict ourselves to the circular cylinder geometry. The analysis of other cylindrical geometries is similar and is expected to yield similarly good results provided that the pinned-end boundary condition is maintained; but of course this conjecture must be checked, specially for non-smooth cross-sections of the cylindrical container. Let us mention here that for the moving contact line case and rectangular containers there is also a discrepancy between calculated and measured values (Henderson \& Miles 1990), which could be due to effects not considered in this paper. We shall consider the limit when the gravitational Reynolds number $C^{-1}=\left(g R^{3}\right)^{1 / 2} / v$ (with $g=$ gravitational acceleration, $R=$ radius of the cylinder and $v=$ kinematic viscosity) is large, as happens to be the case except for quite viscous liquids and/or quite small containers. The gravitational Bond number $B=\rho \mathrm{g} R^{2} / \sigma$ (with $\rho=$ density and $\sigma=$ surface tension) and the slenderness of the cylinder $A=d / R$ (with $d=$ depth of the cylinder) are treated as $O(1)$ parameters. In this limit, the non-dimensional damping rate and frequency are

$$
\text { damping rate }=C^{1 / 2} \omega_{1}+C \omega_{2}+O\left(C^{3 / 2}\right), \quad \text { frequency }=\omega_{0}-C^{1 / 2}\left(\omega_{1}+O\left(C^{3 / 2}\right)\right. \text {. }
$$

Here the $O\left(C^{1 / 2}\right)$ term comes from viscous dissipation in the (oscillatory) Stokes boundary layers near the solid wall and the bottom of the container, and the $O(C)$ term comes from $(a)$ viscous dissipation in the bulk and $(b)$ a first correction of viscous dissipation in the Stokes boundary layers. The leading $O\left(C^{3 / 2}\right)$ neglected term essentially comes from viscous dissipation in the oscillatory boundary layer near the free surface. Now, the main point is that the $O(C)$ term has been systematically neglected because it involves a higher-order effect. This is obviously a good assumption from the asymptotic point of view, provided that $C$ is sufficiently small. Unfortunately $C$ is not 'sufficiently small' in practice, but it is just small (of the order of $10^{-4}$ for water if $R$ is of the order of a few $\mathrm{cm}$ ) and the $O(C)$ term can have a non-negligible effect, as already pointed out by Case \& Parkinson (1957) who considered the simpler case of free contact line without capillary hysteresis. In fact, as we shall see, $\omega_{2} / \omega_{1}$ is frequently fairly large, even for the first mode, and the assumption of neglecting the $O(C)$ effect is good only for extremely small values of $C$. Let us point out here that $\omega_{2} / \omega_{1}$ increases for higher-order modes, as will be checked numerically and explained by asymptotic arguments below. Our two-term approximation of the damping rate shows a good agreement with experiments, as we shall see in $\$ 3$. Notice that there is no correction to the non-dimensional frequency at order $C$; this explains why current approximations for the frequency have been seen to be quite good. Let us also anticipate here that if the effect $(b)$ above is neglected when calculating the $O(C)$ correction then the expression for $\omega_{2}$ is much simpler and frequently gives reasonably good results.

The paper is organized as follows. The coefficients, $\omega_{0}, \omega_{1}$ and $\omega_{2}$, of the asymptotic approximations (1.1) are calculated in $\$ 2$, where the dependence of these coefficients on the mode, the slenderness and the Bond number are also briefly analysed. A comparison with the experimental results by Henderson \& Miles (1994) and some concluding remarks are given in $\$ 3$ and $\$ 4$.

\section{The asymptotic calculation of the damping rate and frequency}

We consider a brimful circular cylinder of radius $R$ and depth $d$, and use $R$ and the gravitational time $(R / g)^{1 / 2}$ to non-dimensionalize length and time in the governing equations and boundary conditions. We use cylindrical coordinates $r, \theta$ and $z$ with the 
origin at the static position of the free surface in the axis of symmetry. If in addition we linearize around the static state and make a normal-mode decomposition of the velocity components, $u, v$ and $w$, the pressure $p$ and the shape of the free surface, $z=f(r, \theta, t)$, as

$$
(u, v, w, p, f)=(U(r, z), \mathrm{i} V(r, z), W(r, z), P(r, z), F(r)) \exp (\Omega t+\mathrm{i} m \theta),
$$

then the following linear eigenvalue problem results:

$$
\begin{gathered}
U r+r^{-1} U-m r^{-1} V+W_{z}=0, \\
\Omega U+P_{r}=C\left[U_{r r}+r^{-1} U_{r}-\left(m^{2}+1\right) r^{-2} U+U_{z z}+2 m r^{-2} V\right], \\
\Omega V+m r^{-1} P=C\left[V_{r r}+r^{-1} V_{r}-\left(m^{2}+1\right) r^{-2} V+V_{z z}+2 m r^{-2} U\right], \\
\Omega W+P_{z}=C\left(W_{r r}+r^{-1} W_{r}-m^{2} r^{-2} W+W_{z z}\right), \\
|U|+|V|+|W|+|P|=\text { bounded as } r \rightarrow 0, \\
U=V=W=0 \text { at } r=1 \quad \text { and } \quad \text { at } z=-A, \\
W-\Omega F=V_{z}+m r^{-1} W=U_{z}+W_{r}=0 \\
\left.\begin{array}{l}
P-F+B^{-1}\left(F^{\prime \prime}+r^{-1} F^{\prime}-m^{2} r^{-2} F\right)=2 C W_{z}
\end{array}\right\} \text { at } z=0, \\
F(1)=0, \\
\int_{0}^{1} F(r) r \mathrm{~d} r=0 \quad \text { if } m=0,
\end{gathered}
$$

Equations (2.1)-(2.4) come from the continuity and momentum equations, and the boundary conditions $(2.6)-(2.8 a)$ result from imposing non-slipping at the solid boundaries, equilibrium of tangential and normal stresses at the free surface and attachment of the contact line at the upper edge of the container respectively. Condition (2.5) is readily seen to be equivalent to imposing smoothness of the pressure and velocity fields (and the shape of the interface) at the axis of symmetry, and the volume-conservation equation $(2.8 b)$ is recalled for convenience; the latter is not really needed if $\Omega \neq 0$ because it is a consequence of $(2.1),(2.5)-(2.6)$ and the first condition (2.7), as is readily seen. The problem depends only on the gravitational Reynolds number, $C^{-1}=\left(g R^{3}\right)^{1 / 2} / v$, the slenderness of the container, $A=d / R$, and the gravitational Bond number, $B=\rho \mathrm{g} R^{2} / \sigma$.

For convenience we consider the linearized Reynolds-Orr energy equation (Drazin \& Reid 1981), which is obtained upon multiplication of (2.2)-(2.4) by $r \bar{U}, r \bar{V}$ and $r \bar{W}$ respectively, integration in $0<r<1,-A<z<0$, integration by parts, substitution of (2.1) and (2.5)-(2.7) and extraction of the real part, to obtain

$$
(\operatorname{Re} \Omega) J_{1}=-J_{2},
$$

where

$$
\begin{aligned}
J_{1}= & \int_{-A}^{0} \int_{0}^{1}\left(|U|^{2}+|V|^{2}+|W|^{2}\right) r \mathrm{~d} r \mathrm{~d} z+\int_{0}^{1}\left[\left(r+m^{2} / B r\right)|F|^{2}+r\left|F^{\prime}\right|^{2} / B\right] \mathrm{d} r \\
J_{2}= & \int_{-A}^{0} \int_{0}^{1}\left(\left|U_{r}\right|^{2}+\left|U_{z}\right|^{2}+\left|V_{r}\right|^{2}+\left|V_{z}\right|^{2}+\left|W_{r}\right|^{2}+\left|W_{z}\right|^{2}\right) r \mathrm{~d} r \mathrm{~d} z \\
& +\int_{-A}^{0} \int_{0}^{1}\left[\left(1+m^{2}\right)\left(|U|^{2}+|V|^{2}\right)+m^{2}|W|^{2}-2 m(U \bar{V}+\bar{U} V)\right] r^{-1} \mathrm{~d} r \mathrm{~d} z \\
& +C \int_{0}^{1}\left(\bar{W} W_{z}+W \bar{W}_{z}\right)_{z=0} r \mathrm{~d} r .
\end{aligned}
$$


Equation (2.9) is a balance between the damping rate of the mechanical energy of the system, $-(\operatorname{Re} \Omega) J_{1}$, and the viscous dissipation rate, $J_{2}$.

We shall consider the limit

$$
C \rightarrow 0
$$

without making at this stage any assumption about $A$ and $B$ (although $A$ and $B^{-1}$ cannot be too small for the analysis below to be valid, as it will be explained at the end of this section).

Notice that (2.1)-(2.8) exhibit two kinds of eigenvalues, which behave as $|\Omega| \sim C$ and $|\Omega| \sim 1$ respectively. As $|\Omega| \sim C$ we obtain hydrodynamic modes that are usually ignored in weakly nonlinear analyses, even though their existence is well-known (e.g. Lamb 1932) and can be seen to be responsible for the viscous secondary streaming flow associated with surface waves; see Nicolás \& Vega (1996) and Nicolás, Rivas \& Vega $(1997,1998)$ for the analysis of these flows in related fluid configurations. Hydrodynamic modes are calculated by seeking solutions of $(2.1)-(2.8)$ of the form

$$
\Omega=C \Omega_{0}+O\left(C^{2}\right), \quad(U, V, W)=\left(U_{0}, V_{0}, W_{0}\right)+O(C), \quad(P, F)=C\left(P_{0}, F_{0}\right)+O\left(C^{2}\right),
$$

to obtain, at leading order, a purely hydrodynamic linear problem (that is omitted for the sake of brevity), without any surface deformation. Thus, these (overdamped) modes can be ignored when considering the linear evolution of the interface associated with the other kind of modes, which are considered now.

As $|\Omega| \sim 1$ we obtain surface modes, which are perturbations of the purely inviscid modes and involve significant deformations of the free surface. Viscous effects are weak in the bulk, except in the oscillatory boundary layers, near the solid walls and the free surface, which have a thickness of the order of $C^{1 / 2}$ (such that inertia and viscous effects are comparable in momentum convervation equations). The eigenvalue $\Omega$, the free-surface deformation, and the pressure and velocity components in the bulk can be expanded as

$$
\begin{gathered}
\Omega=\Omega_{0}+C^{1 / 2} \Omega_{1}+C \Omega_{2}+\cdots, \\
U=U_{0}+C^{1 / 2} U_{1}+C U_{2}+\cdots, \quad V=\cdots, \quad W=\cdots, \quad P=\cdots, \quad F=\cdots,
\end{gathered}
$$

where the expansions for $V, W, P$ and $F$ are similar to that for $U$.

The coefficients of the expansions (2.10)-(2.11) may be calculated in a straightforward manner, as follows. We would substitute (2.10)-(2.11) into (2.1)-(2.5), to obtain a recursive system of equations in the bulk and boundary conditions at the axis of symmetry. The remaining boundary conditions would be obtained from matching conditions with the solution in the oscillatory boundary layers near the solid walls and the free surface. Finally, $\Omega_{0}$ would be obtained by requiring the $O(1)$ problem to possess a non-trivial solution, and $\Omega_{1}$ and $\Omega_{2}$ would be obtained from solvability conditions of the $O\left(C^{1 / 2}\right)$ and $O(C)$ problems. But then we should deal with two main difficulties. First, we would need to consider three terms in the expansions for the solutions in the bulk and in the boundary layers, and this would lead to fairly involved calculations. Secondly, the solution in the bulk exhibits a singularity at the upper edge of the lateral walls (i.e. at $r=1, z=0$ ) that becomes stronger and stronger as we proceed with higher-order terms in the perturbation process; that singularity must be handled carefully at order $C$ to avoid wrong results when applying the solvability condition. That difficulty comes from the fact that the solvability condition involves an integration-by-parts step that fails at $O(C)$ due to the above-mentioned singularity. The difficulty was first encountered by Ursell (1952) and is always present at contact lines. A first solution was given by Mei \& Liu (1973) upon a careful analysis of a 
thin viscous region near the contact line. Higuera et al. (1994) solved the difficulty by removing the singularity from the $O(C)$ problem before applying the solvability condition, and Nicolás \& Vega (1996), in a more subtle weakly-nonlinear setting. introduced an integral solvability condition that also solved the first difficulty. Here, we shall use that integral condition, which may be seen as either $(a)$ a mathematical generalization of the Reynolds-Orr energy equation (2.9) or $(b)$ the result of adding up the solvability conditions at all asymptotic orders $\left(C^{1 / 2}, C, \ldots\right)$. Since that condition is directly obtained from both the original unperturbed problem (2.1)-(2.8) and the $O$ (1) problem (see (2.12)-(2.15) below), which exhibit a quite weak singularity near the contact line, the integration-by-parts step (which is also needed to obtain the condition) does not fail. Notice that both difficulties would be also avoided if using the energy equation, as Henderson \& Miles (1994) did, but then only the real part of $\Omega$ would be obtained and we would not ensure that the $O(C)$ correction in $(2.10)$ is real.

We shall only need the first two terms in the expansions (2.11), which are given by

$$
\begin{aligned}
& U_{0 r}+r^{-1} U_{0}-m r^{-1} V_{0}+W_{0 z}=\Omega_{0} U_{0}+P_{0 r}=\Omega_{0} V_{0}+m r^{-1} P_{0}=\Omega_{0} W_{0}+P_{0 z}=0 \\
& \left|U_{0}\right|+\left|V_{0}\right|+\left|W_{0}\right| \text { bounded at } r=0, \quad U_{0}=0 \text { at } r=1, \quad W_{0}=0 \quad \text { at } z=-A, \\
& W_{0}-\Omega_{0} F_{0}=P_{0}-F_{0}+B^{-1}\left(F_{0}^{\prime \prime}+r^{-1} F_{0}^{\prime}-m^{2} r^{-2} F_{0}\right)=0 \quad \text { at } z=0 \\
& \qquad F_{0}(1)=0 \\
& \qquad \int_{0}^{1} F_{0}(r) r \mathrm{~d} r=0 \quad \text { if } m=0 \\
& U_{1 r}+r^{-1} U_{1}-m r^{-1} V_{1}+W_{1 z}=0, \\
& \Omega_{0} U_{1}+P_{1 r}+\Omega_{1} U_{0}=\Omega_{0} V_{1}+m r^{-1} P_{1}+\Omega_{1} V_{0}=\Omega_{0} W_{1}+P_{1 z}+\Omega_{1} W_{0}=0 \\
& \left|U_{1}\right|+\left|V_{1}\right|+\left|W_{1}\right| \text { bounded at } r=0, \quad U_{1}=U_{0 r} /\left(\Omega_{0}\right)^{1 / 2} \quad \text { at } r=1 \\
& W_{1}=-W_{0 z} /\left(\Omega_{0}\right)^{1 / 2} \quad \text { at } z=-A, \\
& W_{1}-\Omega_{0} F_{1}-\Omega_{1} F_{0}=P_{1}-F_{1}+B^{-1}\left(F_{1}^{\prime \prime}+r^{-1} F_{1}^{\prime}-m^{2} r^{-2} F_{1}\right)=0 \quad \text { at } z=0 \\
& F_{1}(1)=0 \\
& \int_{0}^{1} F_{1}(r) r \mathrm{~d} r=0 \quad \text { if } m=0
\end{aligned}
$$

The boundary conditions at $r=1, z=-A$ and $z=0$, in (2.13)-(2.14) and (2.18)(2.20), are obtained from matching conditions with the Stokes boundary layers near the solid walls and the boundary layer near the interface; the solution of $(2.1)-(2.8)$ in these boundary layers is given in the Appendix ((A 1)-(A 12)), where the linear eigenvalue problem (2.12)-(2.15) is also solved in a semi-analytical form.

Now, the integral solvability condition is obtained as follows. Multiply (2.2)-(2.4) by $r U_{0}, r V_{0}$ and $r W_{0}$ respectively, and the last three equations in (2.12) by $-r U,-r V$ and $-r W$ respectively, add, integrate in $0<r<1$ and $-A<z<0$, integrate by parts, replace the continuity equations in (2.1) and (2.12), and the boundary conditions $(2.5)-(2.8)$ and $(2.13)-(2.15)$, and integrate by parts again to obtain

$$
\left(\Omega-\Omega_{0}\right) I_{1}=-I_{2}-I_{3},
$$


where

$$
\begin{aligned}
I_{1}= & -\int_{-A}^{0} \int_{0}^{1}\left(U_{0} U+V_{0} V+W_{0} W\right) r \mathrm{~d} r \mathrm{~d} z+\int_{0}^{1}\left[\left(r+m^{2} / B r\right) F_{0} F+r F_{0}^{\prime} F^{\prime} / B\right] \mathrm{d} r \\
= & -\left(1+\Omega / \Omega_{0}\right) \int_{0}^{1}\left(F P_{0}\right)_{z=0} r \mathrm{~d} r \\
I_{2}= & -C \int_{-A}^{0} \int_{0}^{1}\left(U_{0 r} U_{r}+U_{0 z} U_{z}+V_{0 r} V_{r}+V_{0 z} V_{z}+W_{0 r} W_{r}+W_{0 z} W_{z}\right) r \mathrm{~d} r \mathrm{~d} z \\
& -C \int_{-A}^{0} \int_{0}^{1}\left[\left(1+m^{2}\right)\left(U_{0} U+V_{0} V\right)+m^{2} W_{0} W-2 m\left(U_{0} V+U V_{0}\right)\right] r^{-1} \mathrm{~d} r \mathrm{~d} z \\
& -C \int_{0}^{1}\left(W_{0} W_{z}+W W_{0 z}\right)_{z=0} r \mathrm{~d} r \\
= & -C \int_{0}^{1}\left(W_{0} W_{z}+3 W W_{0 z}\right)_{z=0} r \mathrm{~d} r \\
I_{3}= & C \int_{-A}^{0}\left(V_{0} V_{r}+W_{0} W_{r}\right)_{r=1} \mathrm{~d} z-C \int_{0}^{1} r\left(U_{0} U_{z}+V_{0} V_{z}\right)_{z=-A} \mathrm{~d} r
\end{aligned}
$$

The second expression for $I_{1}$ in (2.23) is readily obtained upon multiplication of the last three (momentum) equations in (2.12) by $r U, r V$ and $r W$ respectively, integration in $0<r<1,-A<z<0$, integration by parts and application of the continuity equation in (2.12) and the boundary conditions $(2.13)-(2.15 a)$. In order to obtain the second expression for $I_{2}$ first notice that since the inviscid vector field $\boldsymbol{v}_{0}=$ $\left(U_{0} \boldsymbol{e}_{r}+\mathrm{i} \boldsymbol{V}_{0} \boldsymbol{e}_{\theta}+\boldsymbol{W}_{0} \boldsymbol{e}_{z}\right) \mathrm{e}^{\Omega t+\mathrm{i} m \boldsymbol{\theta}}$ is irrotational, its Laplacian, $\nabla^{2} \boldsymbol{v}_{0}=\nabla\left(\nabla \cdot \boldsymbol{v}_{0}\right)-\nabla \times\left(\nabla \times \boldsymbol{v}_{0}\right)$ vanishes and thus $\Delta U_{0}-\left(m^{2}+1\right) r^{-2} U_{0}+2 m r^{-2} V_{0}=\Delta V_{0}-\left(m^{2}+1\right) r^{-2} V_{0}+2 m r^{-2} U_{0}=$ $\Delta W_{0}-m^{2} r^{-2} W_{0}=0$, where $\Delta \varphi=\varphi_{r r}+r^{-1} \varphi_{r}+\varphi_{z z}$. Then we only need to multiply these three equations by $r U, r V$ and $r W$, integrate, integrate by parts and take into account the boundary conditions $(2.13)-(2.15)$.

In order to get some insight into the physical meaning of (2.22), a comparison with the mechanical energy equation (2.9) is now made. Since $\mathrm{i} U_{0}, \mathrm{i} V_{0}, \mathrm{i} W_{0}, F_{0}$ and $P_{0}$ are real, $I_{1}$ and $I_{2}$ are also real and coincide with $J_{1}$ and $J_{2}$ in first approximation, provided that the contribution from the boundary layers is ignored. Thus $I_{1}$ and $I_{2}$ give respectively the mechanical energy and the rate of viscous dissipation in the bulk in first approximation. The latter is given by

$$
-4 C \int_{0}^{1} W_{0}(1, z) W_{0 z}(1, z) r \mathrm{~d} r+C \int_{-A}^{0} V_{0}(1, z)^{2} \mathrm{~d} z
$$

as readily obtained by first ignoring the contribution of the boundary layers and replacing $(U, V, W)$ by $\left(U_{0}, V_{0}, W_{0}\right)$ in the first expression for $I_{2}$ and then proceeding as in the derivation of the second expression for $I_{2}$ above. Thus (2.26) gives the rate of viscous dissipation in the bulk in first approximation, and the difference between that expression and $I_{2}$ gives an $O(C)$ part of the viscous dissipation in the Stokes boundary layers; the remaining $O\left(C^{1 / 2}\right)+O(C)$ parts are given by $I_{3}$.

If the expansion (2.10) and the solutions in the boundary layers (A 1)-(A 3), (A 5)(A 7) and (A 9)-(A 11) are substituted into (2.22) the following expressions for $\Omega_{1}$ and 
$\Omega_{2}$ are obtained:

$$
\begin{aligned}
2 \Omega_{0}^{3 / 2} \Omega_{1} \int_{0}^{1} F_{0}(r) P_{0}(r, 0) r \mathrm{~d} r & =\int_{-A}^{0}\left(m^{2} P_{0}^{2}+P_{0 z}^{2}\right)_{r=1} \mathrm{~d} z \\
& +\int_{0}^{1}\left(r P_{0 r}^{2}+m^{2} P_{0}^{2} / r\right)_{z=-A} \mathrm{~d} r \\
\left(\Omega_{2}+\Omega_{1}^{2} / \Omega_{0}\right) \int_{0}^{1} F_{0}(r) P_{0}(r, 0) r \mathrm{~d} r=-\int_{0}^{1}\left[2\left(r F_{0}^{\prime} P_{0 r}+m^{2} r^{-1} F_{0} P_{0}\right)+\Omega_{1} r F_{1} P_{0}\right]_{z=0} \mathrm{~d} r & \\
& +\int_{-A}^{0}\left[2\left(\Omega_{0}\right)^{1 / 2}\left(m^{2} P_{0} P_{1}+P_{0 z} P_{1 z}\right)+m^{2} P_{0}^{2}-P_{0: z}^{2}\right]_{r=1} \mathrm{~d} z /\left(4 \Omega_{0}^{2}\right) \\
& +\int_{0}^{1}\left(m^{2} r^{-1} P_{0} P_{1}+r P_{0 r} P_{1 r}\right)_{z=-1} \mathrm{~d} r /\left(2 \Omega_{0}^{3 / 2}\right)
\end{aligned}
$$

In order to obtain (2.27)-(2.28), it is convenient to use the second expressions for $I_{1}$ and $I_{2}$ and to notice that (the boundary of the domain is inside the boundary layers and thus) the velocity components $U, V$ and $W$ in the line integrals must be taken from the solution in (A 1)-(A 12). Notice that we are ignoring two corner tori near the edges of the bottom of the container and the free surface $(r=1, z=-A$ and 0$)$, with a characteristic size of the order of $C^{1 / 2}$; the contribution of these tori (where the gradients of $U_{0}, V_{0}, W_{0}, U, V$ and $W$ are $O(\log C)$-quantities) is readily seen to provide $O(C \log C)^{2}$-terms in the expansion for $\Omega$.

Now, the coefficient $\Omega_{1}$ is readily calculated when the expressions for $F_{0}$ and $P_{0}$ in (A 16)-(A 17) are substituted into (2.27). That expression coincides (up to a change in notation) with its comterpart already calculated by Henderson \& Miles (1994), as readily seen when using the semi-analytical expressions for the integrals given in the Appendix ((A 22)-(A 25)). Notice that since $F_{0}$ and $P_{0}$ are real and $\Omega_{0}$ is purely imaginary, $(2.27)$ shows that $\Omega_{1}=-(1+i) \omega_{1}$, with $\omega_{1}$ real and positive (as is well known). In order to apply (2.28) to calculate $\Omega_{2}$ we need to solve the non-homogeneous singular linear problem (2.16)-(2.21), which has a solution if and only if the forcing terms satisfy a solvability condition; that condition is readily seen to coincide with (2.27) (and thus, it is automatically satisfied when $\Omega_{1}$ is calculated as above). A semi-analytical solution to $(2.16)-(2.21)$ is given in the Appendix, in (A 18)-(A 21). Notice that $F_{1} /\left(\Omega_{0}\right)^{1 / 2}$ and $P_{1} /\left(\Omega_{0}\right)^{1 / 2}$ are real and thus (2.28) shows that $\Omega_{2}$ is real, as anticipated above.

A simpler 'approximate' expression for $\Omega_{2}$ is obtained when ignoring the $O\left(C^{1 / 2}\right)$ correction of $I_{1}$ and the $O(C)$ correction of $I_{3}$ and approximating $I_{2}$ by $(2.26)$, to obtain

$$
\begin{aligned}
\tilde{\Omega}_{2} \int_{0}^{1} F_{0}(r) P_{0}(r, 0) r \mathrm{~d} r= & -2 \int_{0}^{1}\left(r F_{0}^{\prime} P_{0(r}+m^{2} r^{-1} F_{0} P_{0}\right)_{z=0} \mathrm{~d} r \\
& -m^{2} \int_{-A}^{0} P_{0}(1, z)^{2} \mathrm{~d} z / 2 \Omega_{0}^{2} .
\end{aligned}
$$

Notice that the approximation $\Omega \simeq \Omega_{0}+C^{1 / 2} \Omega_{1}+C \tilde{\Omega}_{2}$ essentially corresponds to considering only the leading-order approximations of the mechanical energy and the rate of viscous dissipation in the bulk and in the Stokes boundary layers. Still, a comparison of (2.29a) and (2.27) shows that if the last term on the right-hand side of $(2.29 a)$ is ignored then the error in our approximation above is not larger than $\left|\Omega_{1}\right| C /\left|\Omega_{0}^{1 / 2}\right|$; thus the term can be safely ignored except perhaps for small values of 
$\left|\Omega_{0}\right|$ (i.e. for small values of $A$, as explained below). If that term is ignored, then $\tilde{\Omega}_{2}$ is approximated by

$$
\check{\Omega}_{2}^{*} \int_{0}^{1} F_{0}(r) P_{0}(r, 0) r \mathrm{~d} r=-2 \int_{0}^{1}\left(r F_{0}^{\prime} P_{0 r}+m^{2} r^{-1} F_{0} P_{0}\right)_{z=0} \mathrm{~d} r
$$

Notice that the approximation $\Omega \sim \Omega_{0}+C^{1 / 2} \Omega_{1}+C \tilde{\Omega}_{2}^{*}$ corresponds to considering only the leading-order approximations of $I_{1}, I_{2}$ and $I_{3}$ when applying (2.22). These two approximations will be checked in $\$ 3$ where we shall see that they are frequently (but not always) reasonably good. The integrals appearing in $(2.29 a)$ and $(2.29 b)$ are calculated in closed-form in the Appendix ((A 22)-(A 23), (A 26)). For the sake of brevity we omit the associated (quite involved) closed-form expressions for the remaining integrals (involving $F_{1}$ and $P_{1}$ ) appearing on the right-hand side of (2.28).

Let us now consider the limiting values of the slenderness, the eigenfrequency and the Bond number:

(a) A straightforward asymptotic analysis shows that, for a fixed mode,

$$
\left|\Omega_{0}\right| / A^{1 / 2} \rightarrow c_{0}, \quad\left|\Omega_{1}\right| A^{3 / 4} \rightarrow c_{1} \quad \text { and } \quad\left|\Omega_{2}\right| A^{2} \rightarrow c_{2} \quad \text { as } A \rightarrow 0,
$$

where $c_{0}, c_{1}$ and $c_{2}$ are non-zero. Our asymptotic analysis above requires the thickness of the oscillatory boundary layers, $\left(C /\left|\Omega_{0}\right|\right)^{1 / 2}$, to be small compared to the slenderness $A$, or, according to $(2.30)$,

$$
A \gg C^{2 / 5} \text {. }
$$

If that condition does not hold then viscous effects cannot be ignored in the bulk; but, since $A$ is small, a lubrication approximation can be used that greatly simplifies the original three-dimensional problem (2.1)-(2.8) (to a limiting, two-dimensional problem). Notice that if

$$
C^{2 / 5} \ll A \ll 1,
$$

then $C\left|\Omega_{2}\right| \sim C / A^{2} \ll C^{1 / 2} / A^{3 / 4} \sim C^{1 / 2}\left|\Omega_{1}\right|$. This suggests that if (2.31) holds but $A$ is small then the leading-order approximation of the damping rate should provide good results for the first few modes (but not for higher-order modes, according to remark (c) below); but even in this case, it is advisable to calculate the $O(C)$ correction to obtain a better approximation.

(b) As $A \rightarrow \infty, \Omega_{0}, \Omega_{1}$ and $\Omega_{2}$ converge to their limiting values that are obtained upon substitution of $\tanh \left(\lambda_{n} A\right)$ by 1 in (A 15) and (A 20), and substitution of $\cosh \left[\lambda_{n}(z+A)\right] / \cosh \left(\lambda_{n} A\right)$ by $\exp \left(\lambda_{n} z\right)$ in (A 17) and (A 19). Since that convergence is exponential, the limit gives quite good results for moderately large values of $A$, as will be checked in $\$ 3$.

(c) For higher-order modes $\left|\Omega_{0}\right| \rightarrow \infty$ and the eigenfunctions are surface waves with a large wavenumber $k$, such that $\left|\Omega_{0}\right|^{2} \sim k+B^{-1} k^{3}$, and decay exponentially as $k z \rightarrow-\infty$. The validity of the analysis above requires the thickness of the boundary layer attached to the interface, $\left(C /\left|\Omega_{0}\right|\right)^{1 / 2}$, to be small compared to the thickness of the inviscid layer affected by the waves, $k^{-1}$, that is

$$
C \ll\left|\Omega_{0}\right| / k^{2} \sim\left[k^{-3}+(B k)^{-1}\right]^{1 / 2} .
$$

If that condition does not hold then viscous effects must be considered everywhere in the thin layer affected by the waves. If $(2.33)$ holds but $\left|\Omega_{0}\right|$ is large then the viscous dissipation in the Stokes boundary layer attached to the bottom of the container is exponentially small; the viscous dissipation in the boundary layer attached to the 


$\begin{array}{ccccccccccc}(m, q) & \omega_{0} & \omega_{1} & \omega_{2} & \tilde{O}_{2} & \sigma_{2} & \omega & C^{1 / 2} \omega_{1} \times 10^{3} & \delta \times 10^{3} & \delta \times 10^{3} & \tilde{\delta}^{*} \times 10^{3} \\ (1,0) & 1.561 & 0.494 & 13.29 & 11.30 & 11.48 & 1.557 & 4.11 & 5.04 & 4.89 & 4.91 \\ (2,0) & 2.120 & 0.636 & 32.04 & 28.75 & 29.02 & 2.115 & 5.30 & 7.52 & 7.29 & 7.31 \\ (0,1) & 2.287 & 0.187 & 38.21 & 36.93 & 36.93 & 2.285 & 1.56 & 4.21 & 4.12 & 4.12 \\ (3,0) & 2.614 & 0.734 & 56.64 & 52.15 & 52.47 & 2.608 & 6.11 & 10.05 & 9.73 & 9.77 \\ (1,1) & 2.868 & 0.280 & 70.74 & 69.11 & 69.14 & 2.866 & 2.33 & 7.24 & 7.13 & 7.13 \\ (4,0) & 3.099 & 0.812 & 86.73 & 81.11 & 81.45 & 3.092 & 6.76 & 12.78 & 12.39 & 12.42\end{array}$

TABLE 1. The coefficients $\omega_{0}, \omega_{1}, \omega_{2}, \tilde{\sigma}_{2}$ and $\tilde{\omega}_{2}^{*}$ and the approximations of the non-dimensional eigenfrequency and damping rate of the ( $m, q)$ mode (with $m$ nodal diameters and $q$ nodal circles), for $A=1.374, B=103.8$ and $C=6.94 \times 10^{-5}$.

lateral wall and in the bulk are readily seen to be proportional to $k^{-1}\left(\left|\Omega_{0}\right| C\right)^{1 / 2}$ and to $k C$ respectively, and thus

$$
C^{1 / 2}\left|\Omega_{1}\right| /\left(C\left|\Omega_{2}\right|\right) \sim\left[\left|\Omega_{0}\right| /\left(C k^{4}\right)\right]^{1 / 2} \ll k^{-1} \ll 1 .
$$

Then the $O(C)$ correction of the damping rate is much higher than the $O\left(C^{1 / 2}\right)$ leading term as $k$ (or $\left|\Omega_{0}\right|$ ) increases, for a fixed value of $C$. This will be numerically checked in $\$ 3$ and explains why the discrepancy with experiments of the $O\left(C^{1 / 2}\right)$ approximation by Henderson \& Miles (1994) increased for higher-order modes.

(d) As $B \rightarrow \infty$ capillary effects are confined to a $O\left(B^{-1 / 2}\right)$ capillary boundary layer near the edge of the interface. The validity of the analysis above requires the thickness of this layer to be large compared to the thickness of the viscous boundary layers, $\left(C /\left|\Omega_{0}\right|\right)^{1 / 2}$, that is

$$
B C \ll\left|\Omega_{0}\right| .
$$

If $(2.35)$ does not hold then capillary and viscous effects must be considered simultaneously in a $O\left(\left(C /\left|\Omega_{0}\right|\right)^{1 / 2}\right)$ corner torus near the edge of the free surface. Notice that when considering the problem as nearly inviscid in the capillary boundary layer (as we did above) the underlying small parameter is $B C /\left|\Omega_{0}\right|$, which is much larger than $C$ if $B$ is large.

\section{Comparison with experiments}

According to the results in $\$ 2$, the dimensional eigenfrequency $\omega$ and damping rate $\delta$ are given by

$$
\omega=\omega_{0}-C^{1 / 2} \omega_{1}+O\left(C^{3 / 2}\right), \quad \delta=C^{1 / 2} \omega_{1}+C \omega_{2}+O\left(C^{3 / 2}\right)
$$

where

$$
\omega_{0}=\Omega_{0} / \mathrm{i}, \quad \omega_{1}=-\Omega_{1} /(1+\mathrm{i}) \quad \text { and } \omega_{2}=-\Omega_{2} .
$$

Here $\Omega_{0}, \Omega_{1}$ and $\Omega_{2}$ are as calculated from the characteristic equation (A 13) and the expressions (2.27) and (2.28), with $F_{0}, P_{0}, F_{1}$ and $P_{1}$ as given by (A 16)-(A 19). In addition, we suggested the following approximations for the damping rate:

$$
\begin{aligned}
& \tilde{\delta}=C^{1 / 2} \omega_{1}+C \tilde{\omega}_{2} \quad \text { with } \tilde{\omega}_{2}=-\tilde{\Omega}_{2}, \\
& \tilde{\delta}^{*}=C^{1 / 2} \omega_{1}+C \tilde{\omega}_{2}^{*} \quad \text { with } \tilde{\omega}_{2}^{*}=-\tilde{\Omega}_{2}^{*} \text {, }
\end{aligned}
$$

where $\tilde{\Omega}_{2}$ and $\tilde{\Omega}_{2}^{*}$ are given by $(2.29 a, b)$. Those approximations are much simpler because they do not require calculating $P_{1}$ and $F_{1}$.

For illustration, in table 1 we consider several modes for $A=1.372, B=103.8$ 

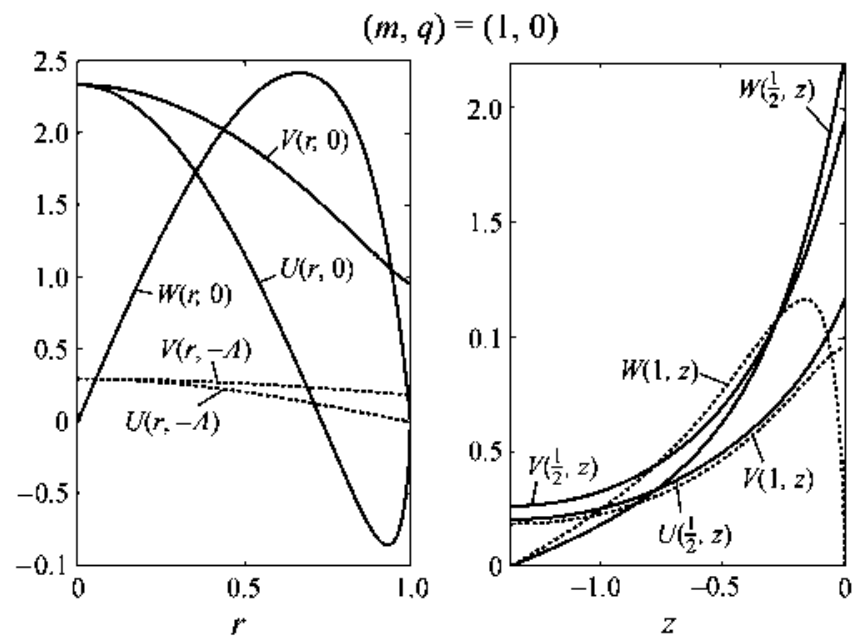

$(m, q)=(0,1)$
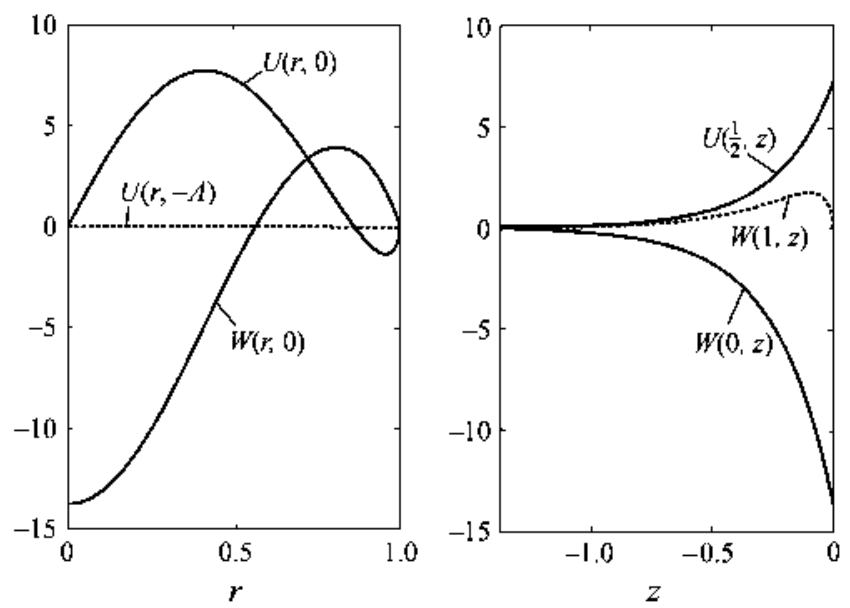

FIGURE 1. Re-scaled non-dimensional components of the velocity, $U=\Omega_{0} U_{0}, V=\Omega_{0} V_{0}$ and $W=\Omega_{0} W_{0}$, at the edge of the Stokes boundary layers (---) and in the bulk (- - , for the $(1,0)$ and $(0,1)$ modes.

and $C=6.94 \times 10^{-5}$ (precisely the values corresponding to the experiment considered below). Several remarks about this table are in order:

(a) If the actual slenderness is replaced by $A=\infty$ then the numerical results in table 1 essentially stand, as anticipated at the end of $\$ 2$.

(b) The ratio $\omega_{2} / \omega_{1}$ is fairly large as anticipated above. According to our discussion in $\S 2$, the underlying reason must be that the Stokes boundary layers attached to the solid walls are quite weak. This is illustrated in figure 1, where we plot the tangential velocities at the edge of the Stokes boundary layers and the velocities in the bulk, for the $(1,0)$ and $(0,1)$ modes; in fact we are plotting the real quantities $\Omega_{0} U_{0}, \Omega_{0} V_{0}$ and $\Omega_{0} W_{0}$. Notice that the tangential velocities at the edge of the Stokes boundary layers (which control viscous dissipation there) are small compared to the gradients of the velocities in the bulk. 


\begin{tabular}{ccccccccccc}
$(m, q)$ & $\omega_{0}$ & $\omega_{1}$ & $\omega_{2}$ & \multicolumn{1}{c}{$\tilde{\sigma}_{2}$} & \multicolumn{1}{c}{$\tilde{\sigma}_{2}$} & $\omega$ & $C^{1 / 2} \omega_{1} \times 10^{3}$ & $\delta \times 10^{3}$ & $\tilde{\delta} \times 10^{3}$ & $\tilde{\delta}^{*} \times 10^{3}$ \\
$(1,0)$ & 0.943 & 1.813 & 17.71 & 8.22 & 8.58 & 0.928 & 15.1 & 16.3 & 15.67 & 15.69 \\
$(0,1)$ & 1.851 & 1.657 & 41.74 & 34.32 & 34.32 & 1.837 & 13.8 & 16.7 & 16.18 & 16.18
\end{tabular}

TABLE 2 . As in table 1 but with $A=0.2$.

\begin{tabular}{|c|c|c|c|c|c|c|c|c|c|c|}
\hline \multirow[b]{2}{*}{$(m, q)$} & \multicolumn{4}{|c|}{ This paper } & \multicolumn{2}{|c|}{ Experiment } & \multicolumn{4}{|c|}{ Approximation by $\mathrm{H} \& \mathrm{M}$} \\
\hline & $f_{0}$ & $f$ & 4 & $\boldsymbol{A}_{E} / \Delta$ & $f_{E}$ & $\Delta_{E}$ & $f_{0}$ & $f$ & $\Delta$ & $\Delta_{E} / \Delta$ \\
\hline$(1,0)$ & 4.68 & 4.67 & 1.37 & 1.02 & 4.65 & 1.4 & 4.68 & 4.66 & 1.13 & 1.2 \\
\hline$(2,0)$ & 6.35 & 6.34 & 1.75 & 1.03 & 6.3 & 1.8 & 6.35 & 6.32 & 1.24 & 1.4 \\
\hline$(0,1)$ & 6.85 & 6.85 & 0.95 & 1.26 & 6.84 & 1.2 & 6.75 & 6.73 & 0.44 & 2.7 \\
\hline$(3,0)$ & 7.84 & 7.82 & 2.11 & 1.04 & 7.80 & 2.2 & 7.84 & 7.79 & 1.29 & 1.7 \\
\hline$(1,1)$ & 8.60 & 8.59 & 1.45 & 1.03 & 8.57 & 1.5 & 8.60 & 8.57 & 0.48 & 3.1 \\
\hline$(4,0)$ & 9.30 & 9.27 & 2.47 & 0.97 & 9.26 & 2.4 & 9.30 & 9.24 & 1.32 & 1.8 \\
\hline
\end{tabular}

TABlE 3. Comparison with Henderson \& Miles (1994) (H \& M) experiments and theoretical predictions for the modes considered in table $1 ; f$ is the dimensional frequency (in c.p.s.) and $\boldsymbol{A}$ is a non-dimensional damping rate (with the non-dimensionalization by $\mathrm{H} \& \mathrm{M}$ ).

(c) The ratio $\omega_{2} / \omega_{1}$ increases as the order of the mode increases, as anticipated at the end of $\$ 2$.

(d) As a consequence of (b) the leading-order approximation of the damping rate is quite poor. This can no longer be true when the slenderness is significantly decreased (for fixed values of $B$ and $C$, and a fixed mode), as explained in $\$ 2$. In order to illustrate this statement we consider the case $A=0.2$ in table 2 .

(e) The approximations $\hat{\delta}$ and $\tilde{\delta}^{*}$ are quite good, for the values of the parameters considered (and for some others we have checked) as anticipated in $\$ 2$. But these approximations could not be so good for other values of the parameters, specially for small $A$ and not-so-small values of $C$. This is illustrated in table 2 where we see that $\tilde{\omega}_{2}$ and $\tilde{\omega}_{2}^{*}$ do not approximate $\omega_{2}$ well. Since $C$ is quite small in this table, $\tilde{\delta}$ and $\tilde{\delta}^{*}$ provide reasonably good approximations of $\delta$, but the approximations worsen as $C$ increases; for instance, if $C=10^{-3}$ then $\tilde{\delta}$ and $\tilde{\delta}^{*}$ are only within $15 \%$ of $\delta$ for the $(0,1)$ mode.

In order to compare with the experiments by Henderson \& Miles (1994) we must consider the dimensional frequency

$$
f=(g / R)^{1 / 2}(\omega / 2 \pi) \text { c.p.s. }
$$

Also, they used a different non-dimensional damping rate $\Delta$, which is related to ours by

$$
\Delta=2\left[g R /\left(\pi v f_{0}\right)\right]^{1 / 2} \delta
$$

where $f_{0}$ is their calculated value of the dimensional inviscid frequency, which is tabulated in column 8 of table 3 . Their experiment was made with pure water (density $\rho=1 \mathrm{~g} \mathrm{~cm}^{-3}$, surface tension $\sigma=72.4 \mathrm{dyn} \mathrm{cm}^{-1}$ and kinematic viscosity $v=0.01 \mathrm{~cm}^{2} \mathrm{~s}^{-1}$ ) in a brimful cylindrical container of radius $R=2.766 \mathrm{~cm}$ and depth $d=3.80 \mathrm{~cm}$, which give the values of $A, B$ and $C$ considered above. As we see in table 3, there is a discrepancy between our calculated value of the inviscid frequency $f_{0}$ of the $(0,1)$ mode and that calculated by Henderson \& Miles (1994); since, as explained in the Appendix, our solution to the $O(1)$ problem is identical to 
theirs, the discrepancy is seemingly due to a material mistake in the computations. Consequently, there is also a significant discrepancy in the viscous approximation of the eigenfrequency for that mode; the slight discrepancy for the remaining modes is due to the fact that they approximated the eigenfrequency as $f=f_{0}-\gamma_{E}$, where $\gamma_{E}$ is the measured dimensional damping rate in c.p.s. Notice that the approximation of the damping rate in this paper is quite good (errors are seemingly within the experimental accuracy) except for the $(0,1)$ mode, which is only within $26 \%$ of the measured value. After checking repeatedly our calculations we think that the discrepancy is due to the neglected $O\left(C^{3 / 2}\right)$ terms. We have checked that the discrepancy is not due to (the leading-order approximation of) viscous dissipation in the oscillatory boundary layer attached to the interface, the only physical effect that was neglected in our approximation. Thus the discrepancy should be due to a higher-order approximation of effects already considered. This could appear as unlikely at first sight, if it is assumed that the relative error of our approximation of each physical effect is of the order of $C^{1 / 2} \sim 10^{-2}$; but, as explained at the end of $\$ 2$ (remark $(d)$ ), the relative error is really of the order of $\left(C B /\left|\Omega_{0}\right|\right)^{1 / 2} \sim 10^{-1}$. Notice that this error decreases as $\left|\Omega_{0}\right|$ increases and thus our approximation should be increasingly good for higherorder modes; but, unfortunately, we do not have experimental results at hand for comparison.

\section{Concluding remarks}

The asymptotic approximations of the eigenfrequency and damping rate derived above apply as $C \rightarrow 0$ provided that the restrictions (2.31), (2.33) and (2.35) hold. Our results explain why the already-known two-term approximation of the eigenfrequency are quite good because the $O(C)$ correction vanishes. We have greatly improved the current one-term approximation of the damping rate by including a $O(C)$ correction that essentially includes the effects of viscous damping in the bulk. The approximation shows a satisfactory agreement with experiments except for the first axisymmetric mode; for this mode our result is only within $26 \%$ of the measured value and this discrepancy is seemingly due to still higher order terms that have been neglected.

Our results are expected to also compare reasonably well with experiments for other values of the parameters, provided that $C \ll 1$ and the above-mentioned restrictions hold. Also, the main point in the paper, namely that a second-order approximation is necessary to calculate the damping rate, is expected to apply to other shapes of the cross-section of the container (i.e. rectangular cross-sections), and is known to apply to related surface-wave problems (in e.g. capillary bridges). A precise calculation of damping rates is in turn essential to construct a quantitatively consistent theory of weakly-nonlinear waves.

This research was supported by DGICYT (Grant PB-94-0416), NATO (Grant CGR-97-0032) and NASA (Grant UGS97-0308). We are indebted to the Editor and the anonymous referees for useful comments.

\section{Appendix}

We give here several groups of algebraic expressions that were omitted in $\S 2$, to facilitate the reading of that section. The first group deals with the solution in the oscillatory boundary layers. The velocity components in the Stokes boundary layer 
near $r=1$ are readily found to be as given by

$$
\begin{aligned}
U(\xi, z)= & C^{1 / 2} U_{0 r}(1, z)\left[\xi+(1-\Gamma(\xi)) / \Omega_{0}^{1 / 2}\right]+O(C), \\
V(\xi, z)= & {\left[V_{0}(1, z)+C^{1 / 2} V_{1}(1, z)\right][1-\Gamma(\xi)] } \\
& +C^{1 / 2} V_{0}(1, z)\left[\left(1-\Omega_{1} / \Omega_{0}^{1 / 2}\right) \xi \Gamma(\xi) / 2+\xi\right]+O(C), \\
W(\xi, z)= & {\left[W_{0}(1, z)+C^{1 / 2} W_{1}(1, z)\right][1-\Gamma(\xi)] } \\
& +C^{1 / 2} W_{0}(1, z)\left(1-\Omega_{1} / \Omega_{0}^{1 / 2}\right) \xi \Gamma(\xi) / 2+O(C) .
\end{aligned}
$$

Here, for $j=0$ and $1, U_{j}, V_{j}$ and $W_{j}$ are the velocity components at $O(1)$ and $O\left(C^{1 / 2}\right)$ in the bulk, and the stretched variable $\xi$ and the function $\Gamma$ are

$$
\xi=(r-1) / C^{1 / 2}, \quad \Gamma(\xi)=\exp \left(\Omega_{0}^{1 / 2} \xi\right) .
$$

Similarly, the velocity components in the Stokes boundary layer near $z=-A$ are

$$
\begin{aligned}
U(r, \eta)= & {\left[U_{0}(r,-\Lambda)+C^{1 / 2} U_{1}(r,-A)\right][1-\Gamma(-\eta)] } \\
& +C^{1 / 2} \Omega_{1} U_{0}(r,-A) \eta \Gamma(-\eta) / 2 \Omega_{0}^{1 / 2}+O(C), \\
V(r, \eta)= & {\left[V_{0}(r,-A)+C^{1 / 2} V_{1}(r,-A)\right][1-\Gamma(-\eta)] } \\
& +C^{1 / 2} \Omega_{1} V_{0}(r,-A) \eta \Gamma(-\eta) / 2 \Omega_{0}^{1 / 2}+O(C), \\
W(r, \eta)= & C^{1 / 2} W_{02}(r,-\Lambda)\left[\eta-(1-\Gamma(-\eta)) / \Omega_{0}^{1 / 2}\right]+O(C),
\end{aligned}
$$

where the function $\Gamma$ is as defined in (A 4) and the stretched variable $\eta$ is

$$
\text { eta }=(z+A) / C^{1 / 2} .
$$

Finally, the velocity components and the pressure in the interface boundary layer are

$$
\begin{aligned}
U(r, \zeta)= & U_{0}(r, 0)+O\left(C^{1 / 2}\right), \quad V(r, \zeta)=V_{0}(r, 0)+O\left(C^{1 / 2}\right) \\
W(r, \zeta)= & \Omega_{0} F_{0}+C^{1 / 2}\left[\Omega_{0} F_{1}+\Omega_{1} F_{0}+\zeta W_{0 z}(r, 0)\right]+O(C), \\
P(r, \zeta)= & F_{0}-B^{-1}\left(F_{0}^{\prime \prime}+r^{-1} F_{0}^{\prime}-m^{2} r^{-2} F_{0}\right) \\
& +C^{1 / 2}\left[F_{1}-B^{-1}\left(F_{1}^{\prime \prime}+r^{-1} F_{1}^{\prime}-m^{2} r^{-2} F_{1}\right)+\zeta P_{0 z}(r, 0)\right]+O(C),
\end{aligned}
$$

in terms of the stretched variable

$$
\zeta=z / C^{1 / 2} .
$$

In the second group of expressions we give the solution of the (inviscid) linear eigenvalue problem (2.12)-(2.15). That problem has a non-trivial solution if, and only if, the following characteristic equation holds:

$$
a_{0}+\sum_{n=1}^{\infty} a_{n} /\left(\mathbf{1}+\lambda_{n}^{2} / B\right)=1,
$$

where $\lambda_{1}, \lambda_{2}, \ldots$ are the strictly positive roots of

$$
J_{m}^{\prime}\left(\lambda_{n}\right)=0
$$


and the coefficient $a_{n}$ is given by

$$
\begin{array}{llr}
a_{0}=-2 I_{0}^{\prime}\left(B^{1 / 2}\right) /\left[B^{1 / 2} I_{0}\left(B^{1 / 2}\right)\right] & \text { if } m=0, \quad a_{0}=0 \quad \text { if } m \geqslant 1, & \text { (A 15a) } \\
a_{n}=2 \Omega_{0}^{2} \lambda_{n}^{2} I_{m}^{\prime}\left(B^{1 / 2}\right) /\left[B^{1 / 2} I_{m}\left(B^{1 / 2}\right)\left(m^{2}-\lambda_{n}^{2}\right)\left(\Omega_{0}^{2}+\lambda_{n}\left(1+\lambda_{n}^{2} / B\right) \tanh \left(\lambda_{n} A\right)\right)\right] & \text { if } n \geqslant 1 .
\end{array}
$$

(A 15b)

Here $J_{m}$ and $I_{m}$ are the $m$ th Bessel and modified Bessel functions respectively. All solutions of (A 13) are seen to be such that $\Omega_{0}^{2}<0$ (i.e. $\Omega_{0}$ is purely imaginary). When (A 13) holds, the shape of the interface $F_{0}$ and the pressure $P_{0}$ are given (up to an arbitrary non-zero complex factor) by

$$
\begin{aligned}
& F_{0}=I_{m}\left(B^{1 / 2} t^{\prime}\right) / I_{m}\left(B^{1 / 2}\right)+a_{0}+\sum_{n=1}^{\infty} a_{n} J_{m}\left(\lambda_{n} r^{t}\right) /\left[\left(1+\lambda_{n}^{2} / B\right) J_{m}\left(\lambda_{n}\right)\right] \\
& P_{0}=a_{0}+\sum_{n=1}^{\infty} a_{n} J_{m}\left(\lambda_{n} t\right) \cosh \left(\lambda_{n}(z+A)\right) /\left[J_{m}\left(\lambda_{n}\right) \cosh \left(\lambda_{n} A\right)\right] .
\end{aligned}
$$

The velocity components $U_{0}, V_{0}$ and $W_{0}$ are readily obtained from (A 17) and the momentum conservation equations in (2.12). This semi-analytical solution coincides with that given by Henderson \& Miles (1994) except for notation. Our solution is equivalent (as seen after several manipulations) to that obtained by Graham-Eagle (1983) for the axisymmetric case in deep containers $(A \rightarrow \infty)$; the main difference is that his solution converges much slower than that by Henderson \& Miles (1994), and than that above. Let us point out here that a similar fast convergent solution was obtained by Sanz (1985) for a related inviscid problem in the liquid bridge geometry.

In the third group of expressions we give the solutions to the first-order problem (2.16)-(2.21). The shape of the interface $F_{1}$ and the pressure $P_{1}$ are given by

$$
\begin{aligned}
F_{1}=K F_{0} & +\left[r F_{0 r}-2 F_{0}+(A-1) F_{0 A}\right] / \Omega_{0}^{1 / 2} \\
& +\sum_{n=1}^{\infty}\left[B\left(b_{n}+2 c_{n}\right)\left(B+\lambda_{n}^{2}\right)+2 B^{2} a_{n}\right] J_{m}\left(\lambda_{n} r\right) /\left[\Omega_{0}^{1 / 2}\left(B+\lambda_{n}^{2}\right)^{2} J_{m}\left(\lambda_{n}\right)\right], \\
P_{1}=K P_{0} & +\left[r P_{0 r}+z P_{0 z}+(\lambda-1) P_{0 .}\right] / \Omega_{0}^{1 / 2} \\
& +\sum_{n=1}^{\infty} b_{n} J_{m}\left(\lambda_{n} r\right) \cosh \left[\lambda_{n}(z+A)\right] /\left[\Omega_{0}^{1 / 2} J_{m}\left(\lambda_{n}\right) \cosh \left(\lambda_{n} A\right)\right],
\end{aligned}
$$

where $K$ is an arbitrary constant, the subscripts $r, z$ and $A$ stand for partial derivatives as above, and the coefficients $a_{n}, b_{n}$ and $c_{n}$ are given by (A15) and

$$
\begin{aligned}
& {\left[\Omega_{0}^{2}+\lambda_{n}\left(1+\lambda_{n}^{2} / B\right) \tanh \left(\lambda_{n} A\right)\right] b_{n}} \\
& \quad=\Omega_{0}^{2}\left[3+2(A-1) \Omega_{0 A} / \Omega_{0}-2 \Omega_{1} / \Omega_{0}^{1 / 2}-2 B /\left(B+\lambda_{n}^{2}\right)\right]\left[a_{n}+c_{n}\left(1+\lambda_{n}^{2} / B\right)\right], \\
& c_{n}=2 B^{1 / 2} \lambda_{n}^{2} I_{m}^{\prime}\left(B^{1 / 2}\right) /\left[I_{m}\left(B^{1 / 2}\right)\left(\lambda_{n}^{2}+B\right)\left(\lambda_{n}^{2}-m^{2}\right)\right],
\end{aligned}
$$

if $n \geqslant 1$. The velocity components $U_{1}, V_{1}$ and $W_{1}$ are readily calculated from (2.17) and (A 19). Let us point out here that when (A 18)-(A 19) are substituted into (2.28) to calculate $\Omega_{2}$, then the resulting value of $\Omega_{2}$ is independent of the arbitrary constant $K$ appearing in (A 18)-(A 19) (as readily seen and required for self-consistency). Also, if $K=0$ then $F_{1} / \Omega_{0}^{1 / 2}$ and $P_{1} / \Omega_{0}^{1 / 2}$ are real (recall that $P_{0}$, 
$F_{0}, a_{n}$ and $\Omega_{1} / \Omega_{0}^{1 / 2}$ are real, and $\Omega_{0}$ is purely imaginary). In order to obtain (A 18)(A 19) we only need to eliminate $U_{1}, V_{1}$ and $W_{1}$ from $(2.16)-(2.21)$ to obtain a linear problem in $P_{1}$ and $F_{1}$, and then to rewrite the problem in terms of the new variables $F^{*}=F_{1}-\left[r F_{0 r}-2 F_{0}+(A-1) F_{0.1}\right] / \Omega_{0}^{1 / 2}$ and $P^{*}=P_{1}-\left[r P_{0 r}+z P_{0 z}+(A-1) P_{0, A}\right] / \Omega_{0}^{1 / 2}$. The new problem satisfies the same equation in the bulk and exhibits non-zero boundary conditions only at the interface $z=0$, and is readily solved in a semianalytical form.

In the fourth group of expressions we give the integrals appearing in (2.27), (2.29a) and $(2.29 b)$ in closed-form as

$$
\begin{aligned}
& 2 \int_{0}^{1} F_{0}(r) P_{0}(r, 0) r \mathrm{~d} r=-\Omega_{0}^{2} \sum_{n=0}^{\infty}\left(\lambda_{n}^{2}-m^{2}\right) \lambda_{n}^{-1} a_{n}^{2} \tanh \left(\lambda_{n} A\right) \\
& \int_{-A}^{0} P_{0}(1, z)^{2} \mathrm{~d} z=\sum_{k, n=0}^{\infty} a_{k} a_{n} \frac{\lambda_{k} \tanh \left(\lambda_{k} A\right)-\lambda_{n} \tanh \left(\lambda_{n} A\right)}{\left(\lambda_{k}^{2}-\lambda_{n}^{2}\right)} \\
& \int_{-A}^{0} P_{0 z}(1, z)^{2} \mathrm{~d} z=\sum_{k, n=0}^{\infty} a_{k} a_{n} \lambda_{k} \lambda_{n} \frac{\lambda_{k} \tanh \left(\lambda_{n} A\right)-\lambda_{n} \tanh \left(\lambda_{k} A\right)}{\left(\lambda_{k}^{2}-\lambda_{n}^{2}\right)} \\
& 2 \int_{0}^{1}\left(r P_{0 r}^{2}+m^{2} P_{0}^{2} / r\right)_{z=-A} \mathrm{~d} r=\Omega_{0}^{4} \sum_{n=0}^{\infty}\left(\lambda_{n}^{2}-m^{2}\right) a_{n}^{2} / \cosh { }^{2}\left(\lambda_{n} A\right) \\
& 2 \int_{0}^{1}\left(r F_{0}^{\prime} P_{0 r}+m^{2} r^{-1} F_{0} P_{0}\right)_{z=0} \mathrm{~d} r=-\Omega_{0}^{2} \sum_{n=0}^{\infty}\left(\lambda_{n}^{2}-m^{2}\right) \lambda_{n} a_{n}^{2} \tanh \left(\lambda_{n} A\right)
\end{aligned}
$$

\section{REFERENCES}

BENJAMIN, T. B. \& URSELL. F. 1954 The stability of the plane surface of a liquid in vertical periodic motion. Proc. R. Soc. Lond. A 225, 505-515.

Case, K. M. \& Parkinson, W. C. 1957 Damping of surface waves in an incompressible fluid. $J$. Fluid Mech. 2, 172-184

DrazIN, P. G. \& ReID, W. H. 1981 Hydrodynamic Stability. Cambridge University Press.

GraHam-EAGLE, J. 1983 A new method for calculating eigenvalues with applications to gravitycapillary waves with edge constraints. Math. Proc. Camb. Phit. Soc. 94, 553-564.

Henderson, D. M. \& Miles, J. W. 1990 Single mode Faraday waves in small cylinders. J. Flifid Mech. 213, 95-109.

Henderson, D. M. \& Miles, J. W. 1994 Surface-wave damping in a circular cylinder with a fixed contact line. J. Fluid Mech. 275, 285-299.

Higuera. H. \& Nicolás. J. A. 1997 Linear non-axisymmetric oscillations of nearly-inviscid liquid bridges. Phys. Fluids 9, 276-285.

Higuera, H. Nicolás, J. A. \& VeGA, J. M. 1994 Linear oscillations of weakly dissipative axisymmetric liquid bridges. Phys. Fluids A 6, 438-450.

Jiang, L., Chao-Lung, T., Perlin, M. \& Shultz, W. 1996 Moderate and steep Faraday waves: instabilities, modulation and temporal asymmetries. J. Fluid Mech. 329, $275-307$.

Lamb, H. 1932 Hydrodynamics. Cambridge University Press.

MeI, C. C. \& LIU, L. F. 1973 The damping of surface gravity waves in a bounded liquid. J. Fluid Mech. 59, 239-256.

Miles, J. W. 1967 Surface-wave damping in closed basins. Proc. R. Soc. Lond. A 297, 459-475.

Miles, J. W. \& Henderson. D. M. 1990 Parametrically forced surface waves. Am. Rev. Fluid. Mech. 22, 143-165.

NiCOLÁs. J. A. \& VEGA. J. M. 1996 Weakly nonlinear oscillations of axisymmetric liquid bridges. $J$. Fluid Mech. 328, 95-128. 
Nicolás. J. A., Rivas. D. \& VEGA, J. M. 1997 The interaction of thermocapillary convection and low-frequency vibration in nearly-inviscid liquid bridges. $Z$. Angew. Math. Phys. 48, 389-423.

Nicolás, J. A., Rivas, D. \& VEGA, J. M. 1998 On the steady streaming flow due to high-frequency vibration in nearly inviscid liquid bridges. J. Fluid Mech. 354, 147-174.

SANZ, A. 1985 The influence of the outer bath in the dynamics of axisymmetric liquid bridges. $J$. Fluid Mech. 156, 101-140.

Stokes, C. G. 1851 On the effect of the internal friction of fluids on the motion of pendulums. Trans. Camb. Phit. Soc. 9, 8-106.

URSELL, F. 1952 Edge waves on a sloping beach. Proc. R. Soc. Lotd. A 214, 79-97.

VAN DoRN, W. G. 1966 Boundary dissipation of oscillatory waves. J. Fluid Mech. 24, 769-779. 